\title{
Immediate colour constancy
}

\author{
David H. Foster*, B. J. Craven $\uparrow$ and Elizabeth R. H. Sale \\ Department of Communication and Neuroscience, Keele University, Staffordshire ST5 5BG, UK
}

(Received 14 October 1991)

\begin{abstract}
Colour constancy is traditionally interpreted as the stable appearance of the colour of a surface despite changes in the spectral composition of the illumination. When colour constancy has been assessed quantitatively, however, by observers making matches between surfaces illuminated by different sources, its completeness has been found to be poor. An alternative operational approach to colour constancy may be taken which concentrates instead on detecting the underlying chromatic relationship between the parts of a surface under changes in the illuminant. Experimentally the observer's task was to determine whether a change in the appearance of a surface was due to a change in its reflecting properties or to a change in the incident light. Observers viewed computer simulations of a row of three Mondrian patterns of Munsell chips. The centre pattern was a reference pattern illuminated by a simulated, spatially uniform daylight; one of the outer patterns was identical but illuminated by a different daylight; and the other outer pattern was equivalent but not obtainable from the centre pattern by such a change in illuminant. Different patterns and different shifts in daylight were generated in each experimental trial. The task of the observer was to identify which of the outer patterns was the result of an illuminant change. Observers made reliable discriminations of the patterns with displays of durations from several seconds to less than $200 \mathrm{~ms}$, and, for one observer, with displays of $1 \mathrm{~ms}$. By these measures, human observers appear capable of colour constancy that is extremely rapid, and probably preattentive in origin.
\end{abstract}

The traditional interpretation of colour constancy refers to the invariant appearance of surface colours under changes in the spectral composition of the light source ${ }^{1,2}$. It is a property of obvious practical significance, and it has a long history of investigation ${ }^{3-9}$. Yet recent quantitative studies ${ }^{10-13}$ have shown that when human observers were required to make matches between coloured patches on surfaces illuminated by different light sources they showed poor colour constancy. When it was obtainable, colour constancy was found to depend on the display geometry, prior knowledge, and psychophysical training of the observer in a way that seems to limit the applicability to human vision of several computational theories of colour constancy ${ }^{6,14-16}$. How, then, should the apparently convincing everyday experience of colour constancy be reconciled with these data on colour matching? We have tested colour constancy using an alternative operational approach which concentrates instead on the ability to detect the underlying chromatic relationship between the coloured patches of a scene as the illuminant changes. As has been argued elsewhere ${ }^{2,17}$, the perception of this invariant relationship rather than the constancy of colour percepts may be the more relevant for biological organisms.

In this study, observers compared Mondrian patterns presented simultaneously in displays of variable duration, from several seconds to $1 \mathrm{~ms}$. (Data on the discrimination of sequentially presented Mondrian patterns have been

* Correspondence to Professor D. H. Foster.

$\dagger$ Present address: Department of Psychology, University of Stirling, Stirling FK9 4LA, UK. reported elsewhere ${ }^{17}$.) Observers were able to make reliable discriminations between patterns in which chromatic relationships were preserved or violated, suggesting that some aspects of human colour constancy are determined by fast, relatively low-level, preattentive visual processes.

\section{Methods}

Apparatus and stimuli

Displays of Mondrian patterns were simulated on an RGB colour graphics system with 8-bit resolution on each gun (Ramtek UK Ltd, Basingstoke, Hampshire, 4660 series) under the control of a computer (Sun Microsystems, Inc., Mountain View, CA, USA, type $3 / 160$ ). The stimuli were displayed on a 19 inch high-resolution $(1280 \times 1024$ pixels $)$ RGB monitor (Manitron Displays Ltd, Sandbach, Cheshire, UK, type CLR2005). The frame rate was $60 \mathrm{~Hz}$ and phosphor decay times were $<1 \mathrm{~ms}$. Calculation of chromaticities and luminances was performed by the computer in the inter-trial intervals. Once loaded into the colour graphics system, the instructions required to display the stimuli were executed without further intervention by the host computer, thereby avoiding the timing uncertainties associated with the multi-tasking operating system. The system was calibrated before and at intervals during the experiment with a photometer and telespectroradiometer, the calibrations of which were in turn traceable to the UK National Physical Laboratory. The monitor was placed in a darkened room and viewed by the subject at $1.35 \mathrm{~m}$.

Each stimulus display consisted of a row of three, 
rectangular, $2 \times 4$ degree, vertically oriented Mondrian patterns, with a centre-to-centre spacing of 2.5 degrees. The background field was dark. The patterns were each composed of 32 randomly positioned patches, selected at random from 1149 of the available 1257 in the Munsell Book of Color ${ }^{18}$. (The Munsell set includes the spectral reflectance functions of many naturally occurring formations ${ }^{14,19}$.) Data for the spectral reflectance functions of the Munsell chips were taken from the principal components analysis by Parkkinen et al. ${ }^{20}$ (also personal communication). The patterns were illuminated by different phases of daylight, the data for the spectral energy distributions of which were taken from the principal components analysis by Judd et al. ${ }^{21}$ The correlated colour temperature of the daylight, also selected at random, ranged from 4400 to $30000 \mathrm{~K}$. The centre pattern was illuminated by a daylight with CIE $x$-coordinate 0.31 . The luminance of the display was such that the equivalent mean illuminance on the patches was approximately 30 lux. A dim fixation cross marked the centre of the screen between trials. The display duration was variable. Different patterns and different daylight shifts were generated in each experimental trial.

The pattern on one side of the centre pattern was identical but illuminated by a different daylight ('illuminant change') and the pattern on the other side was an equivalent pattern but not obtainable from the centre pattern by such an illuminant change ('material change'). In the illuminant-change pattern, the illuminant for all the patches in the pattern was changed by a spatially uniform shift along the daylight locus towards either higher or lower colour temperatures. In the materialchange pattern, for half of the patches selected at random the illuminant was shifted to higher colour temperatures, and for the remaining half to lower colour temperatures. The illuminant-change and material-change patterns occupied the left and right positions with equal probability. Observers had to indicate on each trial which was which. Observers were not given feedback. (In a control experiment when feedback was provided, observer E.S. and a third untrained observer were each able to improve their scores with displays of unlimited duration but not with displays of short duration.) Note that the task could not be performed by an observer comparing the colour of a selected patch in each display: locally the illuminantchange trials were indistinguishable from the materialchange trials.

The ability of subjects to detect a change in the patterns, independent of whether it was an illuminant change or a material change, was determined in a separate control experiment. The stimuli were the same as in the main experiment, but only one of the outer two patterns was altered, either by an illuminant change or by a material change. The observer had to indicate the altered pattern.

In each experiment, at each stimulus duration, the percentage of correct discriminations (or detections) was determined over 36-72 trials for each magnitude of shift in colour temperature, the latter characterized by the corresponding unique projection from the daylight locus onto the $x$-axis in CIE colour space.

\section{Subjects}

There were two subjects, each of whom had normal or corrected-to-normal visual acuity and who had been assessed as colour normal on the Farnsworth-Munsell 100 -Hue test. Both were co-authors of this paper.

\section{Results}

Figure 1 shows percentage correct discrimination as a function of the magnitude of the shift $\Delta x$ in illuminant CIE $x$-coordinate for stimulus displays of durations ranging from unlimited (i.e. until the observer responded) to $<200 \mathrm{~ms}$, and, for one observer, to $1 \mathrm{~ms}$. The smooth curves are quadratic functions of the illuminant shift $\Delta x$ which were subjected to a cumulative normal transformation and fitted to the data by maximum likelihood. Threshold values of $\Delta x$ yielding a performance level of $75 \%$ correct are shown by arrows. Standard errors of the threshold were computed by a bootstrap technique ${ }^{22}$.

A formal data analysis using the empirical logistic transform to linearize the percentage correct scores ${ }^{23}$ confirmed, for each observer, that mean discrimination scores were highly significantly better than chance $(P<0.01)$ at each stimulus duration, and that there was a significant positive linear trend $(P<0.05)$ in the transformed scores with increasing shift $\Delta x$ in illuminant at each stimulus duration.

Figure 2 shows a plot of threshold shift $\Delta x$ in illuminant as a function of display duration for each subject (where thresholds from two additional long-duration displays have been included). There was a highly significant linear decline $(P<0.001)$ in $\log$ threshold with log duration for each observer, and, although mean performances by the two observers differed, the gradients of the declines (in log units) were not significantly different $(-0.12 \pm 0.02$ for E.S. and $-0.15 \pm 0.05$ for B.C.).

Data obtained for percentage correct detection of either an illuminant change or a material change as a function of the magnitude of the shift in illuminant $\Delta x$ were similar to those for percentage correct discrimination in Figure 1, but shifted towards smaller values of $\Delta x$. For stimulus displays of unlimited and $250 \mathrm{~ms}$ duration, threshold values of $\Delta x$ for detection were about 0.8 of those for discrimination.

\section{Discussion}

For successful colour constancy to take place, constraints must be imposed on the spectral composition of the illuminant and on the surface spectral reflectance functions ${ }^{14,15}$. It is obvious that if reflectances and illuminants were chosen arbitrarily at each wavelength colour constancy would be impossible. No assertions are made here about what these constraints might be, other than that the constraints obeyed by real surfaces and natural illuminants are likely to be those to which the visual system is accustomed ${ }^{2}$. Realistic illuminants and realistic reflectance functions were therefore used here. Given these constraints, human vision appears remarkably successful in exploiting them: by the measures used in this study, observers appeared capable of colour constancy that was reliable and extremely rapid. (Although by the design of the experiment observers were unable to make use of local colour cues, they might, in principle, have used a cue such as the 'space-averaged' colour of the patterns. This possibility was rejected in control experiments in which spatially uniform and spatially random shifts in illuminant were present in every trial ${ }^{17}$. Observers were still able to identify reliably illuminant-changed displays.)

Performance in discriminating illuminant changes from 

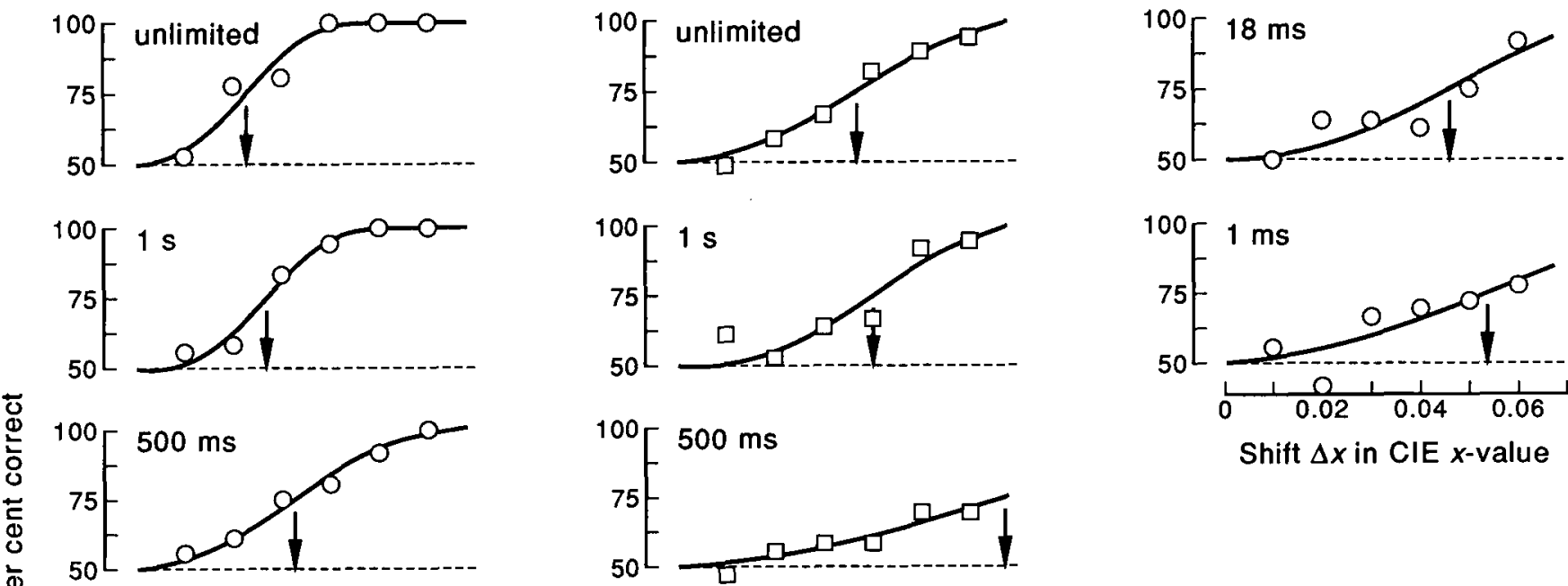

Shift $\Delta x$ in CIE $x$-value
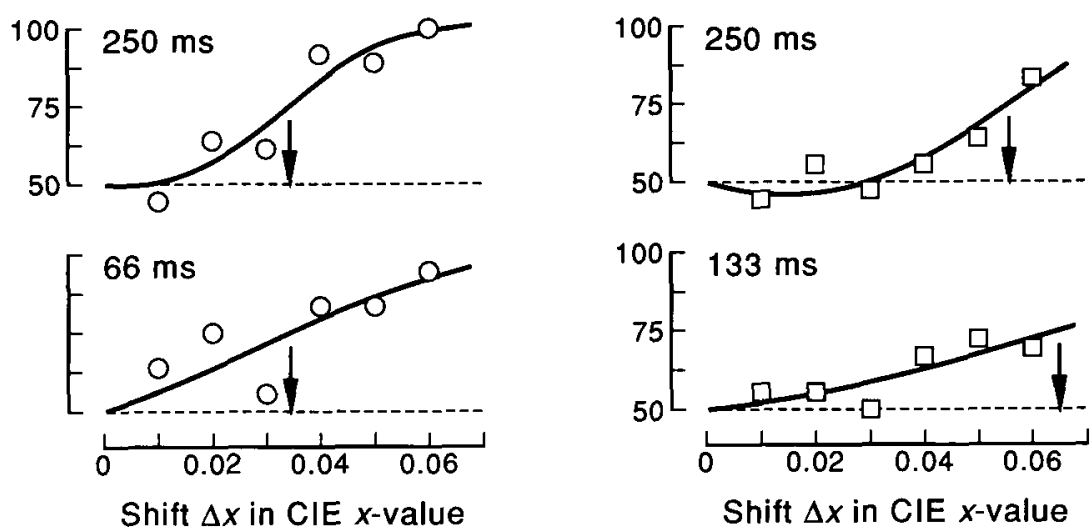

Figure 1 Discrimination of simultaneously presented Mondrian displays subjected to changes in illuminant and material. Performance as percentage correct is plotted as a function of the magnitude of the shift $\Delta x$ in the illuminant CIE $x$-coordinate along the daylight locus. The durations of the displays are shown; the values of $66 \mathrm{~ms}$ and $18 \mathrm{~ms}$ are nominal and correspond respectively to 4 and 2 display cycles at the $60 \mathrm{~Hz}$ frame rate. Each point is based on 36-72 trials. The arrows indicate thresholds for a performance level of $75 \%$ correct. Data for two subjects: $O$, E.S.; $\square$, B.C.

material changes in the Mondrian displays was found to increase steadily with the magnitude of the shift in colour temperature of the illuminant, for all stimulus durations. This result is not trivially predictable, although a simple theoretical rationale has been offered ${ }^{17}$. As the duration of the displays decreased, thresholds for discrimination increased, at similar rates for the two observers. Both observers were able to perform the task with displays of

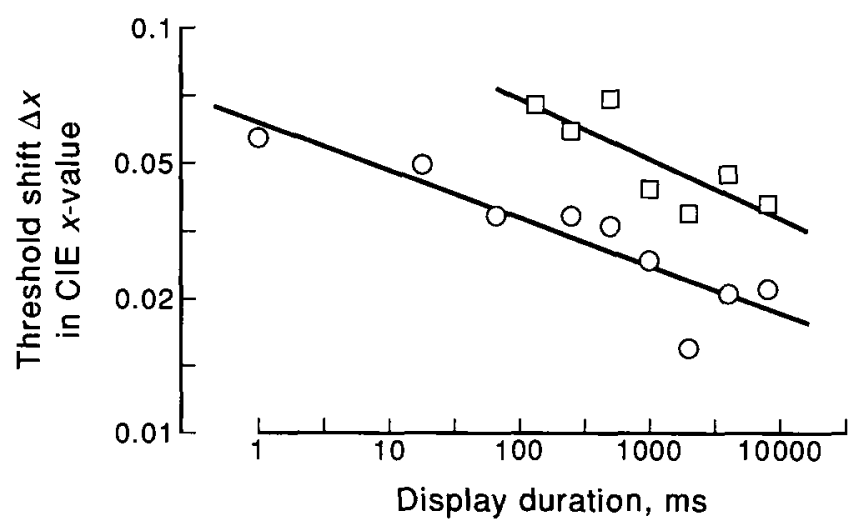

Figure 2. Threshold values of the shift $\Delta x$ in illuminant CIE $x$-coordinate for discriminating illuminant and material changes as a function of display duration. (Standard errors of the threshold values were smaller than the symbol sizes.) Data for two subjects: O, E.S.; $\square$, B.C. less than 200 ms duration, and one observer with displays of $1 \mathrm{~ms}$ duration.

These results, like those of another study of colour constancy in sequentially presented displays ${ }^{17}$, suggest that visual judgements about chromatic relationships may be relatively easily extracted over extended areas without scrutiny of the stimulus, a feature usually associated with preattentive visual processing ${ }^{24,25}$. Although there are several possible mechanisms that could provide a basis for colour constancy ${ }^{26-28}$, it is not entirely clear what effects stimulus exposure and stimulus geometry $^{29}$ would have on their performance or how they should be adapted to the kinds of task considered here.

\section{Acknowledgments}

We are grateful to J. P.S. Parkkinen et al. ${ }^{20}$ for providing characteristic spectra of the Munsell colours. This work was supported by an award from the SERC Image Interpretation Initiative.

\section{References}

1. Boynton, R. M. Human Color Vision. Holt, Rinehart and Winston, New York, USA, pp. 26, 183-185 (1979).

2. Jameson, D. and Hurvich, L. M. Essay concerning color constancy Ann. Rev. Psychol. 40, 1-22 (1989).

3. von Helmholtz, H. Handbuch der Physiologischen Optik, Vol. II, 
3rd Edn (1911). Translation entitled Helmholtz's Treatise on Physiological Optics (ed J. P. C. Southall). Optical Society of America pp. 286-287 (1924) republished by Dover Publications, New York, USA (1962).

4. Hering, E. Collected works (1920) in: Outlines of a Theory of the Light Sense, Translated by L. M. Hurvich and D. Jameson, Harvard University Press, Cambridge, MA, USA, pp. 7-8 (1964).

5. Land, E. H. The Retinex theory of color vision. Sci. Am. 237(6), 108-129 (1977).

6. Land, E. H. Recent advances in Retinex Theory. Vision Res. 26, $7-21(1986)$.

7. McCann, J. J., McKee, S. P., Taylor, T. H. Quantitative studies in Retinex theory: a comparison between theoretical predictions and observer responses to the 'color Mondrian' experiments. Vision Res. 16, 445-458 (1976).

8. Zeki, S. The representation of colours in the cerebral cortex. Nature 284, 412-418 (1980).

9. Andrews, P. R. and Campbell, F. W. Is there more to the Land two-colour effect than colour constancy? (Unpublished results) (1991).

10. Arend, L. and Reeves, A. Simultaneous color constancy. J. Opt. Soc. Am. A 3, 1743-1751 (1986)

11. Reeves, A., Arend, L. E. and Schirillo, J. Color constancy in isolated displays. Perception 18, 529-530 (1989).

12. Tiplitz Blackwell, K. and Buchsbaum G. Quantitative studies of color constancy. J. Opt. Soc. Am. A 5, 1772-1780 (1988).

13. Valberg, A. and Lange-Malecki, B. 'Colour constancy' in Mondrian patterns: a partial cancellation of physical chromaticity shifts by simultaneous contrast. Vision Res. 30, 371-380 (1990).

14. Maloney, L. T. Evaluation of linear models of surface spectral reflectance with small numbers of parameters. J. Opt. Soc. Am. A 3, 1673-1683 (1986).

15. Hurlbert, A. Formal connections between lightness algorithms. J. Opt. Soc. Am. A 3, 1684-1693 (1986).

16. Forsyth, D. A. A novel algorithm for color constancy. Int. J. Comp.
Vision 5, 5-36 (1990).

17. Craven, B. J. and Foster, D. H. An operational approach to colour constancy. Vision Res. (1992) in press.

18. Munsell Book of Color-Matte Finish Collection, Munsell Color, Baltimore, MD, USA (1976).

19. Krinov, E. L. Spectral Reflectance Properties of Natural Formations, Laboratoriia Aerometodov. Akad. Nauk SSSR, Moscow (1947). Translated by G. Belkov, National Research Council of Canada, Ottawa (technical translation: TT-439) (1953).

20. Parkkinen, J. P. S., Hallikainen, J. and Jaaskelainen, T. Characteristic spectra of Munsell colors. J. Opt. Soc. Am. A 6, 318-322 (1989).

21. Judd, D. B., MacAdam, D. L. and Wyszecki, G. Spectral distribution of typical daylight as a function of correlated color temperature. J. Opt. Soc. Am. 54, 1031-1040 (1964).

22. Foster, D. H. and Bischof, W. F. Thresholds from psychometric functions: superiority of bootstrap to incremental and probit variance estimators. Psychol. Bull. 109, 152-159 (1991).

23. Cox, D. R. The Analysis of Binary Data. Methuen, London, UK, pp. 76-87 (1970).

24. Julesz, B. Textons, the elements of texture perception, and their interactions. Nature 290, 91-97 (1981).

25. Treisman, A. Preattentive processing in vision. Comput. Vis. Graphics Image Proc. 31, 156-177 (1985).

26. Zeki, S. Colour coding in the cerebral cortex: the responses of wavelength-selective and colour-coded cells in monkey visual cortex to changes in wavelength composition. Neuroscience 9 , 767-781 (1983).

27. D'Zmura, M. and Lennie, P. Mechanisms of color constancy. $J$. Opt. Soc. Am. A 3, 1662-1672 (1986).

28. Foster, D. H. and Snelgar, R. S. Quasi-independence of opponent-colour processes under chromatic adaptation in man. J. Physiol. 377, 42P (1986).

29. McCann, J. J. Local/global mechanisms for color constancy. Die Farbe 34, 275-283 (1987). 